\title{
THE EXISTENCE OF GOLDEN AGES AND STABILITY IN THE TWO-SECTOR MODEL *
}

\author{
EDWIN BURMEISTER
}

I. Introduction, 146. - II. Brief description of the model, 147. - III. The case $s_{\mathrm{W}}=0,148$. - IV. The case $s_{p}=s_{w}=s, 150 .-\mathrm{V}$. Concluding remarks, 152.

\section{INTRODUCTION}

There are several related questions which can be asked in the context of the neoclassical two-sector growth model.

(1) Starting from any initial capital/labor ratio $k^{o}$, will the capital/labor ratio asymptotically approach a unique equilibrium value $k^{*}$ ? Such a system is called stable.

(2) Starting from any initial capital/labor ratio $k^{o}$, will $k^{t}$ (the capital/labor ratio at time $t$ ) asymptotically approach some equilibrium value $k^{*}, k^{* *}, \ldots, k^{*} \ldots{ }^{*}$ ? Such a system is called globally stable.

(3) Given any initial capital/labor ratio $k^{0}$, is it possible to find constant $s_{p}$ and $s_{w}$ (where $s_{p}$ and $s_{w}$ are the average and marginal propensities to save from profit and wage incomes, respectively) such that $\dot{k}=0$ and there exists a Golden Age with $k^{t}=k^{o}$ for all $t$ ?

(4) Is the Golden Age described by (3) stable? I.e., with $\left(s_{p}{ }^{0}\right.$, $s_{w}{ }^{0}$ ) equal to the savings ratios which will maintain the Golden Age path when the capital/labor ratio is initially $k^{o}$, will $k^{t}$ asymptotically converge to $k^{o}$ if initially the capital/labor ratio is not equal to $k^{\circ}$ ?

(5) Is $\left(s_{p}{ }^{0}, s_{w}{ }^{0}\right)$ uniquely determined by $k^{0}$ ?

(6) Given $\left(s_{p}^{o}, s_{w}^{o}\right)$ (which will maintain $k^{t}=k^{o}$ with $\dot{k}=0$ ), does there exist another Golden Age path such that $k^{t}=k^{00} \neq k^{0}$ ?

While questions (1) and (2) have received extensive study, ${ }^{1}$ a

* The author wishes to express his appreciation to the National Science Foundation for support from Research Grants GS-95 and GS-571. He is also indebted to Professor Paul A. Samuelson for many helpful discussions and to Professors A. R. Dobell and Robert M. Solow for comments about an earlier draft.

1. Some of the many papers which discuss questions (1) and (2) are: Hirofumi Uzawa, "On a Two-Sector Model of Economic Growth: II," Review of Economic Studies, XXX (June 1963) ; Emanuel M. Drandakis, "Factor Substitution in the Two-Sector Growth Model," Review of Economic Studies, XXX (Oct. 1963); Ken-ichi Inada, "On a Two-Sector Model of Economic Growth: Comments and a Generalization," Review of Economic Studies, XXX (June 1963). Many of the important theorems are summarized by Edwin Burmeister, "The Role of the Jacobian Determinant in the Two-Sector Model," forthcoming in the International Economic Review. 
rigorous discussion of (3), (4), (5), and (6) does not appear in the literature. The latter questions are of interest, ${ }^{2}$ and in this paper they will be answered and their relationship examined.

\section{Brief Description of the Model ${ }^{3}$}

The subscripts 1 and 2 denote the capital-goods sector and the consumption-goods sector, respectively. The production functions for both sectors are assumed homogeneous of degree one, and thus we may define

$$
y_{i} \equiv Y_{i} / L_{i}=F_{i}\left(K_{i} / L_{i}, 1\right) \equiv f_{i}\left(k_{i}\right), \quad i=1,2,
$$

where $k_{1} \equiv K_{1} / L_{1}$ and $k_{2} \equiv K_{2} / L_{2} .{ }^{4}$ The total malleable capital stock $(K)$ and the total homogeneous labor supply $(L)$ are assumed fully employed; thus $K_{1}+K_{2}=K$ and $L_{1}+L_{2}=L$. Let $\rho$ equal the ratio of labor employed in the capital-goods sector to total labor, i.e., $\rho \equiv L_{1} / L$. The full-employment assumption is expressed by the equation $\rho k_{1}+(1-\rho) k_{2}=k$ where $k$ is equal to the total capital/ labor ratio $K / L$. We also set $\omega$ equal to the wage/rentals ratio $W / R,{ }^{5}$ assume that $\dot{L} / L=\lambda$ ( = an exogenous constant), and define the growth of the capital stock by

$$
\dot{K} \equiv Y_{1}-\mu K
$$

where $\mu$ is the depreciation rate of capital and is independent of use. Finally; let $n \equiv \lambda+\mu$.

Imposing the condition $\dot{k}=0$, the competitive short-run equilibrium conditions for a given value of $k$ are described by

where

$$
\psi^{i}\left(k_{1}, k_{2}, \omega, \rho ; k\right)=0, \quad i=1, \ldots, 4,
$$

$$
\psi^{1}=\frac{f_{1}\left(k_{1}\right)}{f_{1}^{\prime}\left(k_{1}\right)}-k_{1}-\omega=0
$$

2. Joan Robinson is concerned with a similar problem in her paper "Harrod's Knife-edge," in Collected Economic Papers, III (Oxford: Blackwell, 1966), where on p. 52 she writes, "In the discussion aroused by Sir Roy Harrod's Dynamic Economics there has been some confusion between the existence of a possible steady-growth path and its stability." The relationship between the existence of Golden Age growth paths and stability is precisely the subject of this paper, but only in the context of the two-sector model.

3. A more complete description of the model is given by Uzawa, op. cit.

4. To avoid a discussion of corner solutions, we will assume that the Inada conditions (op. cit.), $f_{i}{ }_{i}\left(k_{i}\right)>0, f^{\prime \prime}{ }_{i}\left(k_{i}\right)<0$ for $0<k_{i}<\infty$ and $f^{\prime}{ }_{i}(0)=$ $\infty, f_{i}^{\prime}(\infty)=0, i=1,2$, are satisfied. If the Inada conditions are not satisfied (e.g., if the production function in the capital-goods sector has a constant elasticity of substitution not equal to one), then it may be necessary to replace the equalities in $\psi=0$ (the system of equations below) by appropriate inequalities. The analysis which follows can be easily modified to include such cases. Also see fn. 6 below.

5. $R$ is the gross rental rate for one unit of the capital good, and $W$ is the wage rate. 


$$
\begin{aligned}
\psi^{2} & =\frac{f_{2}\left(k_{2}\right)}{f_{2}^{\prime}\left(k_{2}\right)}-k_{2}-\omega=0 \\
\psi^{3} & =\rho k_{1}+(1-\rho) k_{2}-k=0 \\
\psi^{4} & =\rho f_{1}\left(k_{1}\right)-n k=0 .
\end{aligned}
$$

The equation $\psi^{4}$ expresses the restriction $\dot{k}=0 .^{6}$

Examination of the system $\psi=0$ shows that the point $x \equiv\left(k_{1}\right.$, $\left.k_{2}, \omega, \rho\right)$ is uniquely determined by $k$, i.e., there exists a (vector) function

$$
x=\phi(k) .^{7}
$$

\section{The CASE $s_{w} \equiv 0$}

Consider the case in which $s_{w} \equiv 0$. The capital/labor ratio grows according to the equation

$$
\frac{\dot{k}}{k}=s_{p} f_{1}^{\prime}\left(k_{1}\right)-n, \quad k \neq 0,
$$

6. To derive $\psi$ from the savings equation

$P_{1} Y_{1}=s_{p} R K+s_{w} W L$, observe that the latter can be written as $\rho f_{1}\left(k_{1}\right)=f_{1}^{\prime}\left(k_{1}\right)\left[s_{p} k+s_{w} \omega\right]$. Since $\dot{k}=f^{\prime}{ }_{1}\left(k_{1}\right)\left[s_{p} k+s_{w} \omega\right]-n k$, the condition $\dot{k}=0$ is expressed by $\psi^{4}=0$. Note also that there exists a maximum sustainable capital/labor ratio $\left(k_{M A X}\right)$ which is equal to the root of the equation $f_{1}(k)-n k=0$ obtained by setting $s_{p}=s_{w}=1$. Likewise, when $s_{w} \equiv 0$ there is a maximum sustainable capital/labor ratio $\left(k_{\text {max }}\right)$ which is the root of the equation $f_{1}^{\prime}\left[k_{1}(k)\right]-n=0$ obtained by setting $s_{p}=1$ and $s_{w}=0$.

The Inada conditions assure that $k_{M A X}$ and $k_{\max }$ as defined above exist for any value of $n \equiv \lambda+\mu$ (see fn. 4 above). Otherwise it may be necessary to define $k_{\max }$ and $k_{\max }$ by appropriate inequalities for some values of $n$, and the only Golden Ages may be $k=0$ or $k=\infty$, but such cornersolution cases are not discussed here. (For example, if $f_{1}^{\prime}(0)=\beta$, a finite constant, and if $n>\beta$, then $k_{\Delta \Delta x}=0$, and we have no problem to consider!)

7 . To prove this fact we first note that $\psi=0$ implies the existence of a function $k=k(\omega)$ (see, e.g., Uzawa, op. cit.). It is thus sufficient to prove that $\frac{d \omega}{d k}>0$ always holds so that there exists an inverse function $\omega=\omega(k)$. Let $J$ denote the Jacobian determinant of $\psi=0$, i.e., let

$$
J=\operatorname{det}\left[\frac{\partial\left(\psi^{1}, \ldots, \psi^{4}\right)}{\partial\left(k_{1}, \ldots, \rho\right)}\right] \text {. }
$$

Then for $J \neq 0, \frac{d \omega}{d k}=\frac{N}{J}$ where $N$ is the determinant formed by replacing the third column of $J$ with the column vector $\left[-\frac{\partial \psi^{1}}{\partial k}, \ldots,-\frac{\partial \psi^{4}}{\partial k}\right]=$ $[0,0,1, n]$. Since it is easily shown that both $N$ and $J$ are strictly positive, the conclusion that $x=\phi(k)$ for $0<k<k_{M A X}$ (or $0<k<k_{\max }$ if $s_{w} \equiv 0$ ) follows immediately. Similarly we establish that $d k_{i} / d k>0, i=1,2$.

The latter method of proof is used by Burmeister, op. cit. Alternatively, it is easily seen in this case that the system of equations $\psi=0$ is equivalent to finding that tangency point on the "contract curve" in a box diagram which also lies on the isoquant $\mathrm{Y}_{1}=n \mathrm{~K}$. The Inada conditions assure that there exists one and only one such point. 
and we find $\dot{k}=0$ when

$$
s_{p}=\frac{n}{f_{1}^{\prime}\left(k_{1}\right.} \text {. }
$$

Thus $\left.\quad \frac{d s_{p}}{d k}\right|_{\dot{k}=0}=-\left.n \frac{f_{1}^{\prime \prime}\left(k_{1}\right)}{\left[f_{1}^{\prime}\left(k_{1}\right)\right]^{2}} \frac{d k_{1}}{d k}\right|_{\dot{k}_{k}=0}>0$.

A graph of $s_{p}$ versus $k$ along which $\dot{k}=0$ appears in Figure I where $k_{\text {max }}$ is the maximum sustainable capital/labor ratio under the hypothesis $s_{w}=0$. For any given $k^{0} \leqslant k_{\text {max }}$, we can find a value of $s_{p}$ given by $s_{p}{ }^{o}=g\left(k^{o}\right)$ such that $\dot{k}=0$. Likewise for any given value $s_{p}{ }^{0} \leqslant 1$, there exists one and only one value of $k$ given by

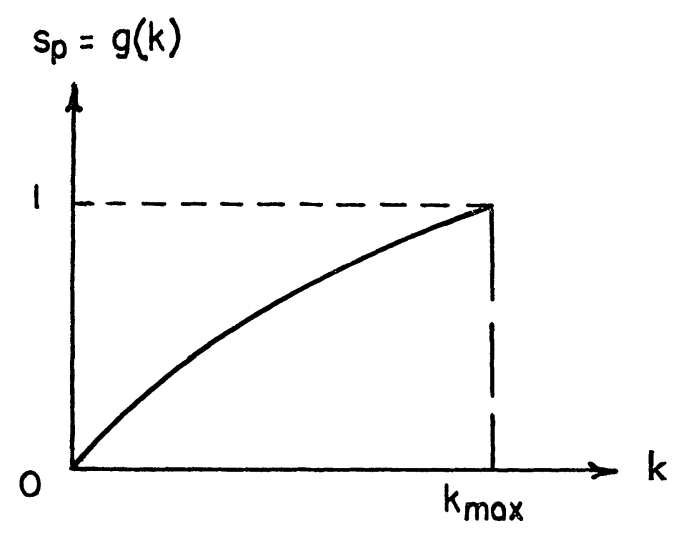

Figure I

$$
k^{o}=g^{-1}\left(s_{p}^{o}\right)
$$

for which $\dot{k}=0$. Thus the answers to questions (3) and (5) are "yes," while the answer to question (6) is "no."

Now suppose we set $s_{p}=s_{p}{ }^{0}$; will $k^{t}$ asymptotically converge to

$$
k^{0}=g^{-1}\left(s_{p}^{o}\right) ?
$$

The answer is "not necessarily," for the system may not be stable in the sense of question (2). More precisely,

$$
\left.\frac{d(\dot{k} / k)}{d k}\right|_{s_{p}{ }^{o}}=\left.s_{p}^{o} f_{1}^{\prime \prime}\left(k_{1}\right) \frac{d k_{1}}{d k}\right|_{s_{p}{ }^{o}}
$$

may be positive since it is well known that $\left.\frac{d k_{1}}{d k}\right|_{s_{p}{ }^{\circ}}$ may be negative. Thus stability implies that the answer to question (4) is 'yes," i.e., if the system is stable, then the Golden Age path is also stable. 
We may summarize our results as follows. If $s_{w}=0$, then:

(1) For any initial $k^{0} \leqslant k_{\text {max }}$, we can find a value $s_{p}{ }^{o}=g\left(k^{0}\right)$ such that $\dot{k}=0$ and $k^{t}=k^{o}$ for all $t$.

(2) For every $s_{p}{ }^{0} \leq 1$, there exists one and only one $k^{0}=$ $g^{-1}\left(s_{p}{ }^{o}\right)$ such that $\dot{k}=0$.

(3) Setting $s_{p}=s_{p}{ }^{0}=g\left(k^{o}\right), k^{t}$ will asymptotically converge to $k^{0}$ starting from any initial value of $k$ if the system is stable. ${ }^{8}$

\section{The CASe $s_{p}=s_{w}=s$}

Now assume that the saving propensities out of profit and wage incomes are equal, i.e., let $s_{p}=s_{\imath 0}=s$, and let $k_{M A X}$ be the maximum sustainable capital/labor ratio. From the equation

$$
\dot{k}=s f_{1}^{\prime}\left(k_{1}\right)(k+\omega)-n k
$$

we find that

$$
\left.s\right|_{\dot{k}=0}=\frac{n k}{f_{1}^{\prime}\left(k_{1}\right)(k+\omega)}
$$

and calculate

$$
\begin{aligned}
& \left.\frac{d s}{d k}\right|_{\dot{k}=0} \\
& f_{1}^{\prime}\left(k_{1}\right)(k+\omega)-k\left\{f_{1}^{\prime}\left(k_{1}\right)\left(1+\frac{d \omega}{d k}\right)+f_{1}^{\prime \prime}\left(k_{1}\right) \frac{d k_{1}}{d k}(k+\omega)\right\} \\
& =n \longrightarrow\left[f_{1}^{\prime}\left(k_{1}\right)(k+\omega)\right]^{2} \quad \mid \dot{k}=0
\end{aligned}
$$

Now the system is stable for any $0<s<1$ if

$$
\frac{d \dot{k}}{d k}=s f_{1}^{\prime}\left(k_{1}\right)\left(1+\frac{d \omega}{d k}\right)+s f_{1}^{\prime \prime}\left(k_{1}\right) \frac{d k_{1}}{d k}(k+\omega)-n<0
$$

or if

$$
f_{1}^{\prime}\left(k_{1}\right)\left(1+\frac{d \omega}{d k}\right)+f_{1}^{\prime \prime}\left(k_{1}\right) \frac{d k_{1}}{d k}(k+\omega)<\frac{n}{s} .
$$

In particular, taking $\dot{k}=0$ or

8. Two sufficient conditions for stability in a two-sector model with $s_{w}=0$ are (1) $k_{1} \leq k_{2}$ or (2) $\sigma_{1}+\sigma_{2} \geqslant 1$ where $\sigma_{\imath}=\frac{\omega}{k_{\imath}} \frac{d k_{i}}{d \omega}$ is the elasticity of substitution in the $i^{\text {th }}$ production function. (See, for example, Drandakis or Burmeister, op. cit.). 


$$
s=\frac{n k}{f_{1}^{\prime}\left(k_{1}\right)(k+\omega)} \text {, it is necessary and sufficient for sta- }
$$

bility that

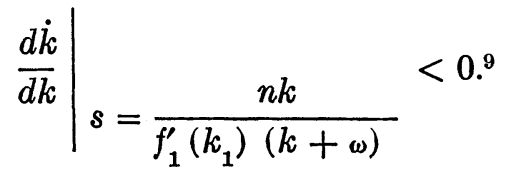

We thus find that stability implies the inequality

$$
k\left[f_{1}^{\prime}\left(k_{1}\right)\left(1+\frac{d \omega}{d k}\right)+f_{1}^{\prime \prime} \frac{d k_{1}}{d k}(k+\omega)\right]<f_{1}^{\prime}\left(k_{1}\right)(k+\omega) .
$$

It follows that

$$
\left.\frac{d s}{d k}\right|_{\dot{k}=0}>n \frac{f_{1}^{\prime}\left(k_{1}\right)(k+\omega)-f_{1}^{\prime}\left(k_{1}\right)(k+\omega)}{\left[f_{1}^{\prime}\left(k_{1}\right)(k+\omega)\right]^{2}}=0 .
$$

Thus if the system is stable, there exists a function $s=h(k)$ for which $\dot{k}=0$ and

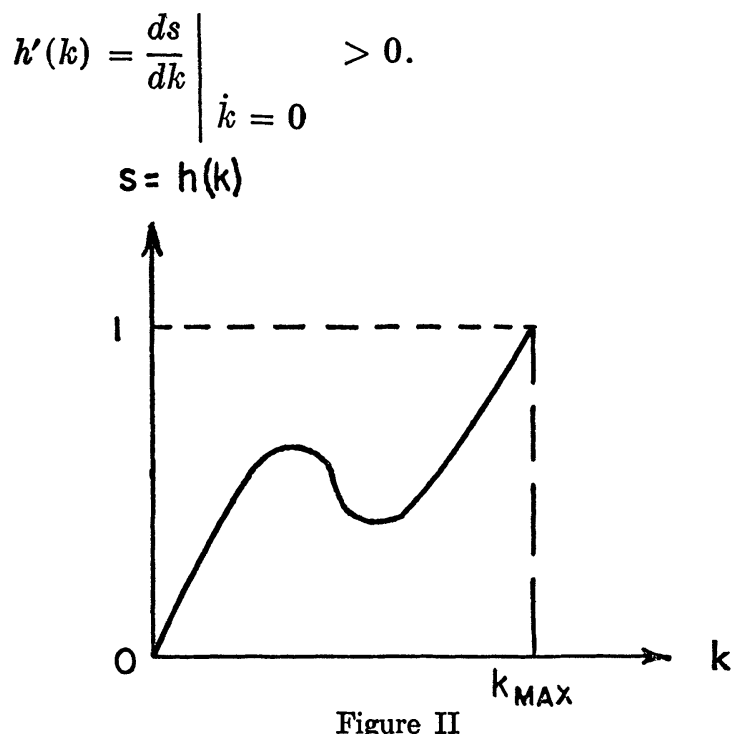

9. Complete rigor requires a qualification of this statement. If we write the growth equation in the form $\dot{k}=f(k)$ and let $k^{*}$ equal a root of $f(k)=0$, then $k^{*}$ is (locally) stable if $f^{\prime}\left(k^{*}\right)<0$. However, suppose that $f(k)$ has an inflection point at $k=k^{*}$ with $f^{\prime}\left(k^{*}\right)=0 ; k^{*}$ is nevertheless a stable root if $f^{\prime}(k)<0$ for all $k \neq k^{*}$. Note, however, that if $k^{*}$ is a stable root, then it is necessary that there exists a neighborhood of $k^{*}$ for which $f^{\prime}(k)<0, k \neq k^{*}$. For this reason the conclusions which follow will not be altered by the existence of such inflection points, and for simplicity we shall rule them out by assumption. 
Even if the system is not stable, the condition $s_{p}=s_{w}=s$ is sufficient for global stability. ${ }^{1}$ In this case the graph of $s$ versus $k$ along which $\dot{k}=0$ may appear, for example, as pictured in Figure II.

We now summarize the results for this case. If $s_{p}=s_{w}=s$ and the system is stable, ${ }^{2}$ then:

(1) For any initial $k^{o} \leq k_{M A X}$, we can find a value $s^{o}=h\left(k^{o}\right)$ such that $\dot{k}=0$ and $k^{t}=k^{o}$ for all $t$.

(2) For every $s^{o} \leq 1$, there exists one and only one $k^{o}=g^{-1}\left(s^{o}\right)$ such that $\dot{k}=0$.

(3) Setting $s_{p}=s^{o}=h\left(k^{o}\right), k^{t}$ will asymptotically converge to $k^{0}$ starting from any initial (positive) value of $k$.

If $s_{p}=s_{w}=s$ and the system is not stable, then:

(1) For any initial $k^{o} \leqslant k_{M A X}$, we can find a value $s^{o}=h\left(k^{o}\right)$ such that $\dot{k}=0$ and $k^{t}=k^{o}$ for all $t$.

(2) $h^{\prime}(k)$ is not of one sign and for some values of $s \leqslant 1$, there may exist more than one value, say $k^{o}, k^{o o}, \ldots$, such that $\dot{k}=0$.

(3) Setting $s=s^{o}=h\left(k^{o}\right), k^{t}$ may or may not asymptotically converge to $k^{0}$ depending on the value of the initial capital/labor ratio; but the system is globally stable, and $k^{t}$ will asymptotically converge to some value. ${ }^{3}$

\section{Concluding Remarks}

What if $s_{p}$ and $s_{w}$ are both positive but $s_{p} \neq s_{w}$ ? Since

$$
s \equiv \frac{P_{1} Y_{1}}{R K+W L}=\frac{s_{p} R K+s_{w} W L}{R K+W L},
$$

$s, s_{p}$, and $s_{w}$ are related by the equation

$$
s(k+\omega)=s_{p} k+s_{w \omega} .
$$

Given a value of the capital/labor ratio $k^{\circ}$, we have shown that there exist unique values $s^{o}$ and $\omega^{o}$ consistent with $k=0$ and $k^{t}=k^{o}$ for all $t$. Thus any values of $s_{p}$ and $s_{w}$ which satisfy

$$
s_{p} k^{0}+s_{w^{\omega}} \omega^{o}=s^{0}\left(k^{0}+\omega^{0}\right)
$$

1. See, for example, Uzawa, op. cit. Thus a system which is only globally stable may (for some fixed values of $s$ ) converge asymptotically to different values $k^{*}, k^{* *}, \ldots, k^{*} \ldots *^{*}$ depending on the initial capital/labor ratio $k^{\circ}$.

2. Two sufficient conditions for stability in a two-sector model with $s_{p}=$ $s_{w}=s$ are (1) $k_{1} \leqslant k_{2}$ or (2) $\sigma_{2} \geqslant 1$. (See Drandakis, Uzawa, and Burmeister, op. cit.).

3. Thus if $s_{p}=s_{w}=s$ and the system is stable, the answers to questions (3), (4), (5), and (6) are "yes," "yes," "yes," and "no," respectively; if the system is not stable, the answers are "yes," "maybe, depending on the initial capital/labor ratio," "yes," and "yes," respectively. 
will imply $\dot{\mathrm{k}}=0$. Clearly, therefore, one can never hope to determine a unique pair $\left(s_{p}, s_{w}\right)$ merely by the condition $\dot{k}=0$; when $0 \neq$ $s_{p} \neq s_{w} \neq 0$, the answer to question (5) is "no."

As to the existence of a Golden Age growth path, note that we are free to set $\left(s_{p}, s_{w}\right)=\left(s^{o}, s^{o}\right)$ where $s^{o}=g\left(k^{o}\right)$. Given any initial capital/labor ratio such that $0<k^{o} \leqslant k_{M A X}$, we can find at least one solution $\left(s_{p}, s_{w}\right)=\left(s^{o}, s^{o}\right)$ such that $\dot{k}=0$ and $k^{t}=k^{o}$ for all $t$; thus question (3) can always be answered "yes." However, as is clear from the above special cases, the answers to questions (4), (5), and (6) depend on the assumptions (if any) about $s_{p}$ and $s_{w}$, the stability of the system, or the initial capital/labor ratio.

Finally, the above analysis can be easily extended to answer a different but related question. It has been observed that some models exhibit "perverse behavior" in which a Golden Age with a low rate of interest (but higher than the Golden Rule value $r=\lambda$ ) does not always correspond to high per capita consumption. ${ }^{4}$ Denoting per capita consumption $\left(Y_{2} / L\right)$ by $C=(1-\rho) f_{2}\left(k_{2}\right)$, we wish to determine the sign of $d C / d r$ in alternative Golden Ages $(\dot{k}=0)$. The system of equations $\psi=0$ already imposes the condition $\dot{k}=0$, and thus it is a simple matter of calculation to find

$$
\left.\frac{d C}{d r}\right|_{\dot{k}=0}=\left.\left[\frac{d C}{d k} \frac{d k}{d r}\right]\right|_{\dot{k}=0}
$$

where in equilibrium the rate of interest $(r)$ is equal to the own-rate of return to capital in the capital-goods sector, i.e.,

$$
r=f_{1}^{\prime}\left(k_{1}\right)-\mu .
$$

It can be shown that

$$
\operatorname{sgn}\left[\left.\frac{d C}{d r}\right|_{\dot{k}=0}\right]=\operatorname{sgn}(\lambda-r),
$$

and thus for all Golden Ages with $r>\lambda(r<\lambda)$, the Golden Age with the lower (higher) rate of interest always has higher per capita consumption. ${ }^{5}$

We conclude that even though the two-sector model may be unstable and may possess multiple equilibria, the behavior of per

4. See Michio Morishima, Equilibrium, Stability, and Growth, (Oxford: Clarendon Press, 1964) p. 126, and Michael Bruno, Edwin Burmeister, and Eytan Sheshinski, "The Nature and Implications of the Reswitching of Techniques," this Journal, LXXX (Nov. 1966).

5. I understand that Professors Robert M. Solow and Michael Bruno have independently obtained the same result 
capita consumption across alternative Golden Ages is not "perverse." The reason the two-sector model cannot exhibit "perverse behavior" is that it contains only one capital good; the conclusion may change if one (or more) different capital good is added. ${ }^{6}$

University of Pennsylvania

6. See Bruno, Burmeister, and Sheshinski, op. cit. 